\title{
CHAPTER 3C.
}

\section{Surveys and Observations: \\ Filaments}




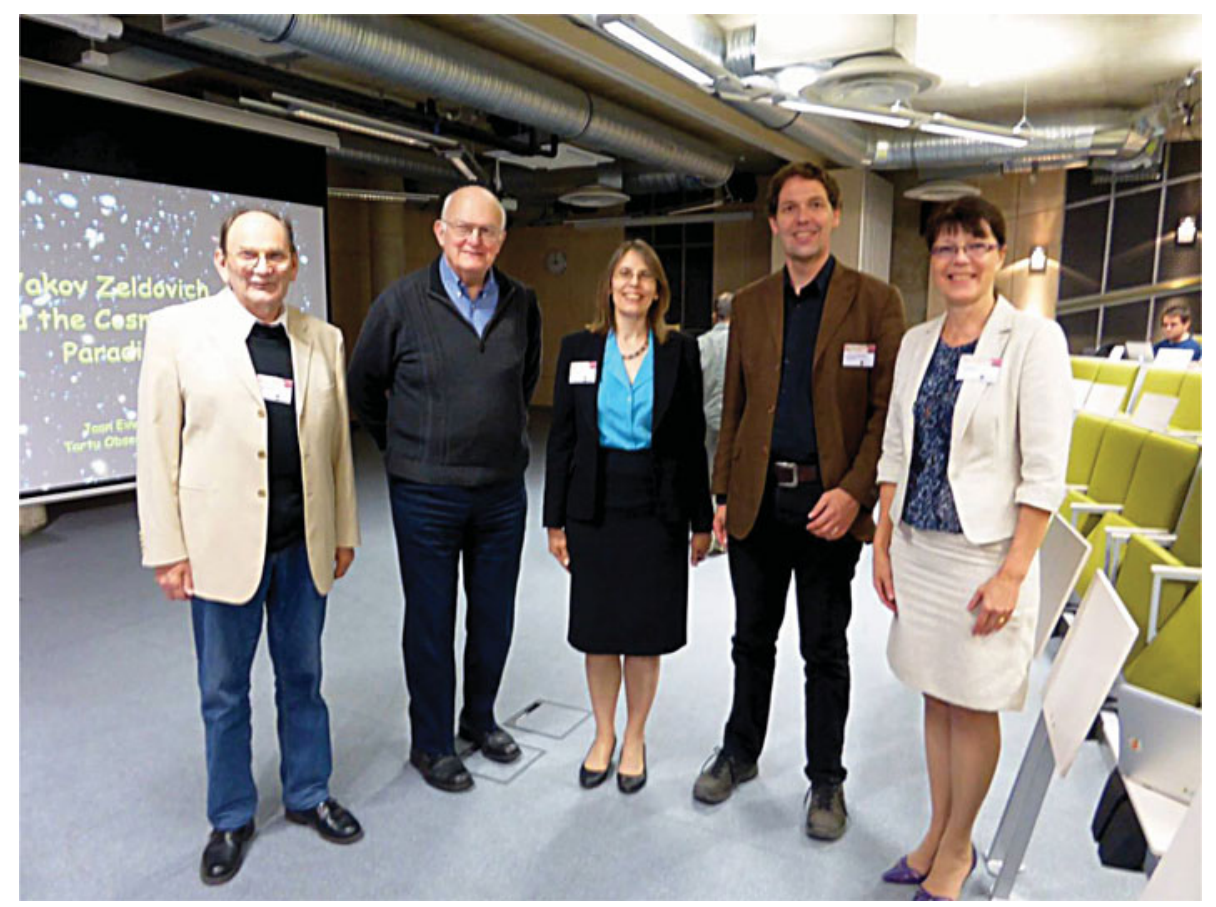

line-up, from left to right:

Jaan Einasto, Alar Toomre, Tiia Lillemaa, Rien van de Weygaert and dr. Anu Reinart, director of Tartu Observatory

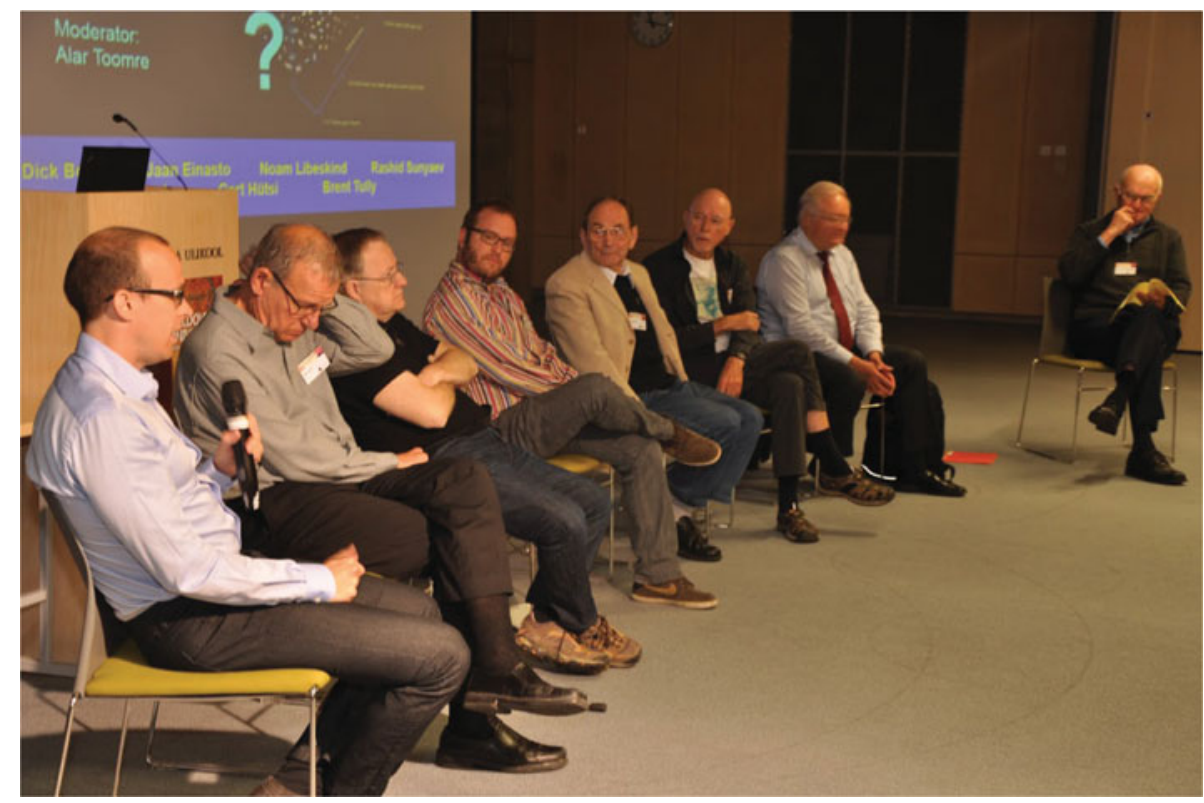

line-up at the Gruber Prize panel discussion on future challenges in cosmology. from left to right: Gert Hütsi, Marc Davis, Dick Bond, Noam Libeskind, Jaan Einasto, Brent Tully, Rashid Sunyaev and Alar Toomre (moderator) 


\title{
Structure and kinematics of the Bootes filament
}

\author{
O. Nasonova ${ }^{1}$, I. Karachentsev ${ }^{1}$ and V. Karachentseva ${ }^{2}$ \\ ${ }^{1}$ Special Astrophysical Observatory of RAS, Nizhnij Arkhyz, Russia \\ ${ }^{2}$ Main Astronomical Observatory of NASU, Kyiv, Ukraine
}

\begin{abstract}
Bootes filament of galaxies is a dispersed chain of groups residing on sky between the Local Void and the Virgo cluster. We consider a sample of 361 galaxies inside the sky area of $\mathrm{RA}=13^{h} .0 \ldots 18^{h} .5$ and $\mathrm{Dec}=-5^{\circ} \ldots+10^{\circ}$ with radial velocities $V_{L G}<2000 \mathrm{~km} / \mathrm{s}$ to clarify its structure and kinematics. In this region, 161 galaxies have individual distance estimates. We use these data to draw the Hubble relation for galaxy groups, pairs as well as the field galaxies, and to examine the galaxy distribution on peculiar velocities. Our analysis exposes the known Virgo-centric infall at RA $<14^{h}$ and some signs of outflow from the Local Void at RA $>17^{h}$. According to the galaxy grouping criterion, this complex contains the members of 13 groups, 11 pairs and 140 field galaxies. The most prominent group is dominated by NGC 5846. The Bootes filament contains the total stellar mass of $2.7 \times 10^{12} M_{\odot}$ and the total virial mass of $9.07 \times 10^{13} M_{\odot}$, having the average density of dark matter to be $\Omega_{m}=0.09$, i.e. a factor three lower than the global cosmic value.
\end{abstract}

Keywords. galaxies: distances and redshifts, cosmology: large-scale structure of universe

\section{Introduction}

Oservational data on distances and velocities of galaxies in the Local Supercluster have been enriched significantly by recent optical and HI surveys, allowing to obtain the nearby field of peculiar motions and hence to study the local distribution of dark matter. Previously, we considered motions of galaxies in some sky areas neighbouring the Virgo cluster as the Local Supercluster centre. In the Virgo Southern Extension filament (Karachentsev \& Nasonova 2013) and the Ursa Majoris cloud (Karachentsev et al. 2013) we derived a low mean matter density: $\Omega_{m}=0.11$ and 0.08 , respectively. But in the Coma I region we suggested the existence of a dark attractor with the total mass of $\sim 2 \times 10^{14} M_{\odot}($ Karachentsev et al. 2011).

\section{Observational data}

The initial sample of galaxies was selected from the Lyon Extragalactic Database = LEDA (http://leda.univ-lyon1.fr) and limited by radial velocities $V_{L G} \leqslant 2000 \mathrm{~km} / \mathrm{s}$ and equatorial coordinates $\mathrm{RA}=13^{h} .0 \ldots 18^{h} .0$ and $\mathrm{Dec}=-5^{\circ} \ldots+10^{\circ}$. The major part of the considered strip is covered by SDSS survey (Abazajian et al. 2009) as well as HIPASS (Zwaan et al. 2003) and ALFALFA (Haynes et al. 2011) HI surveys. It allowed us to perform an independent morphological classification of galaxies and to determine distances for many galaxies from the Tully \& Fisher (1977) relation between luminosity of a galaxy and its $\mathrm{HI}$ line width $W_{50}$. The resulting list of 361 galaxies includes 161 galaxies with individual distance estimates. 

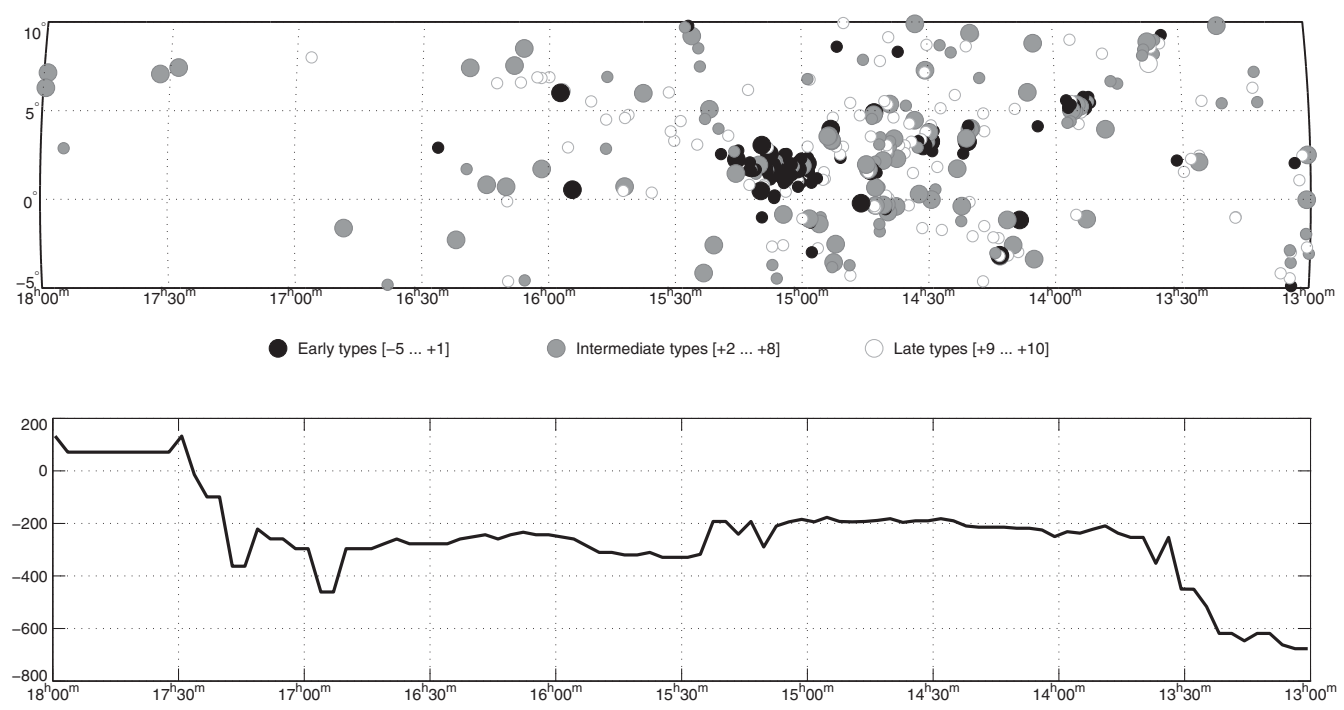

Figure 1. Upper panel: distribution of galaxies in the Bootes strip. Bottom panel: a poliline represents the running median of peculiar velocity with a window of $0^{h} .5$.

\section{Structure and sub-structures}

The Bootes filament of galaxies is a scattered chain of galaxy groups residing between the Local Void $\left(\mathrm{RA}=19^{h} \cdot 0, \mathrm{Dec}=+3^{\circ}\right)$ and the Virgo cluster $\left(\mathrm{RA}=12^{h} .5\right.$, Dec $=+12^{\circ}$ ). The kinematics of this structure should be influenced both by galaxies infalling towards the Virgo cluster as well as more eastern galaxies moving away from the expanding Local Void (Nasonova \& Karachentsev 2011).

The upper panel of Fig. 1 presents the distribution of 361 galaxies in the Bootes strip. The galaxies of different morphological types are shown by circles of different density. According to the galaxy grouping criterion (Makarov \& Karachentsev 2011), the Bootes strip contains 13 groups, 11 pairs and 140 field galaxies. The most notable feature in the strip is the compact group around NGC 5846 (just right from the strip center), which numbers 74 members with measured radial velocities. The bottom panel of Fig. 1 shows the running median of the peculiar velocity, $V_{p e c}=V_{L G}-72 D_{M p c}$, with a window of $0^{h} .5$ along RA. The most common value is $V_{p e c}=-250 \mathrm{~km} / \mathrm{s}$, which remains almost flat from $14^{h}$ to $17^{h}$. Galaxies in the Virgo infall zone $\left(\mathrm{RA}<14^{h}\right)$ demonstrate clearly a droop of the $V_{p e c}$ median. To the contrary, in the vicinity of the Local Void the median rises which is quite expectable since galaxies move away from the void centre.

\section{Local density of matter}

The virial (projected) mass distribution of galaxy groups (squares) and pairs (triangles) in the considered strip versus their total stellar mass is depicted in Fig. 2. There is a positive correlation between virial and stellar masses, well-known from other data. While masses are small, the significant vertical scatter is caused mainly by projection factors.

According to Jones et al.(2006), the mean density of stellar matter in the universe is $4.6 \times 10^{8} M_{\odot} / \mathrm{Mpc}^{3}$. The global matter density $\Omega_{m}=0.28$ is equivalent to dark-to-stellar matter ratio of $M_{D M} / M_{*}=97$. This value is shown as a diagonal in Fig. 2. All the groups and all the pairs except one in the Bootes strip are situated under this line. The Bootes filament contains the total stellar mass of $2.7 \times 10^{12} M_{\odot}$ and the total virial mass of $9.07 \times 10^{13} M_{\odot}$, having the average ratio $\sum M_{p} / \sum M_{*}=33$ or the local density of 


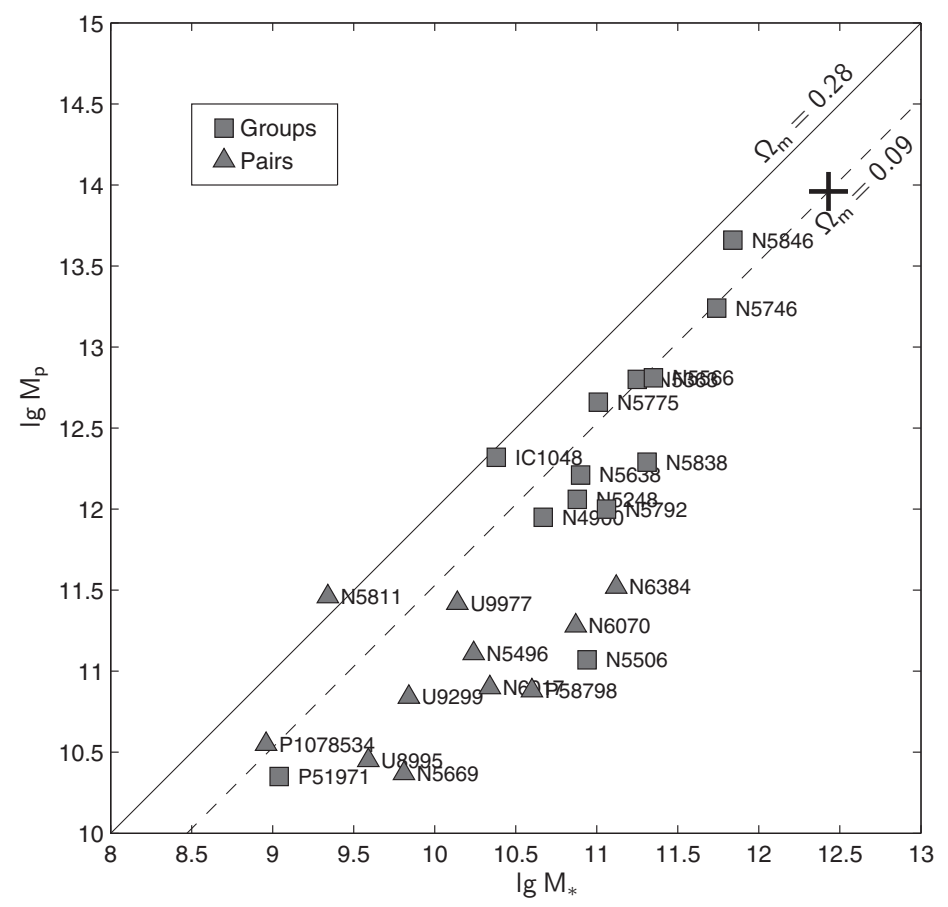

Figure 2. The relation between projected (virial) mass and total stellar mass.

dark matter $\Omega_{m}=0.09$. This is a factor three lower than the global cosmic value. The sum of virial masses-to-sum of stellar masses ratio for all the groups and pairs is plotted in Fig. 2 as a cross. The dashed line drawn through the cross indicates the mean mass density $\Omega_{m}$ (Bootes $) \simeq 0.09$. Considering field galaxies should only reduce slightly this proportion, as they contribute evidently both to numerator and denominator of the ratio $\sum M_{p} / \sum M_{*}$. Thus, the observational data on galaxy motions in the Bootes strip give us an argument that this filamentary structure does not contain a large amount of dark matter. This statement is based on internal (virial) motions of galaxies, but supposing that $3-5$ times larger mass is hidden in the Bootes filament between the groups, then the velocity dispersion for centres of groups and pairs should be considerably larger than what is observed.

This work is supported by the Russian Science Foundation (project No. 14-12-00965), Russian Foundation for Basic Research (grant No. 13-02-90407) and the State Fund for Fundamental Researches of Ukraine (grant No. F53.2/15). Olga Nasonova thanks the non-profit Dmitry Zimins Dynasty Foundation for the financial support. This research has made use of NASA/IPAC Extragalactic Database (http://ned.ipac.caltech.edu), HyperLeda database (http://leda.univ-lyon1.fr) and SDSS archive (http://www.sdss.org).

\section{References}

Abazajian, K. N., Adelman-McCarthy, J. K., Agueros, M. A., et al. 2009, ApJS, 182, 54

Haynes, M.P., Giovanelli, R., Martin, A. M., et al. 2011, AJ, 142, 170

Jones, D. H., Peterson, B. A., Colless, M., \& Saunders, W. 2006, MNRAS, 369, 25

Karachentsev, I. D., Nasonova, O. G., \& Courtois, H. M. 2011, ApJ, 743, 123

Karachentsev, I. D. \& Nasonova, O. G. 2013, MNRAS, 429, 2677 
Karachentsev, I. D., Nasonova, O. G., \& Courtois, H. M. 2013, MNRAS, 429, 2264

Karachentsev, I. D., Tully, R. B., Shaya, E. J., et al. 2014, ApJ, 782, 4

Makarov, D. I. \& Karachentsev, I. D. 2011, MNRAS, 412, 2498

Nasonova, O. G. \& Karachentsev, I. D. 2011, Astrophysics, 54, 1

Tully R. B. \& Fisher R. J. 1977, A\&A, 54, 661

Zwaan, M. A., Staveley-Smith, L., Koribalski, B.S., et al. 2003, AJ, 125, 2842 